\title{
Intertwining operators between some spaces of differential operators on a manifold
}

\author{
P. Mathonet*
}

\begin{abstract}
The Lie algebra of vector fields $\operatorname{Vect}(M)$ of a smooth manifold $M$ acts by Lie derivatives on the space $\mathcal{D}_{\lambda, \mu}^{p}$ of differential operators of order $\leq p$ that map fields of densities of weight $-\lambda$ on fields of densities of weight $-\mu$. In this paper, we determine all the intertwining operators between these modules, when $\operatorname{Dim} M \geq 2$.
\end{abstract}

\section{Introduction}

Let $M$ be a smooth, connected, Hausdorff, second countable manifold of dimension $m$.

We denote by $\Delta^{\lambda}(M)$ the vector bundle of scalar densities of weight $\lambda \in \mathbb{R}$, and by $\mathcal{F}_{\lambda}$ the space of smooth sections of $\Delta^{-\lambda}(M)$. We refer to [2] and [3] for the definitions. Let us recall that for each chart $\left(U,\left(x^{1}, \ldots, x^{m}\right)\right)$ of $M, F \in \mathcal{F}_{\lambda}$ and $X \in \operatorname{Vect}(M)$, the restriction of $F$ over $U$ is of the form

$$
\left.F\right|_{U}=f\left|d x^{1} \wedge \cdots \wedge d x^{m}\right|^{-\lambda},
$$

where $f: U \rightarrow \mathbb{R}$ is smooth. The corresponding local expression of $L_{X}^{\lambda} F$ is then

$$
\left.L_{X}^{\lambda} F\right|_{U}=\left(X . f-\lambda \operatorname{tr}\left(D_{x} X\right) f\right)\left|d x^{1} \wedge \cdots \wedge d x^{m}\right|^{-\lambda}
$$

where $X . f$ is the derivation of $f$ in the direction of $X, \operatorname{tr}$ denotes the trace and $D_{x} X$ is the differential of the local expression of $X$. The Lie derivative on differential operators is given by the commutator : for every differential operator $D: \mathcal{F}_{\lambda} \rightarrow \mathcal{F}_{\mu}$ and $X \in \operatorname{Vect}(M)$,

$$
L_{X}^{\lambda, \mu} D=L_{X}^{\mu} \circ D-D \circ L_{X}^{\lambda}
$$

is a differential operator of order at most that of $D$. Equipped with (2), the space of differential operators of order $\leq p$ from $\mathcal{F}_{\lambda}$ into $\mathcal{F}_{\mu}$ is then a module over $\operatorname{Vect}(M)$, which we will denote by $\mathcal{D}_{\lambda, \mu}^{p}$.

${ }^{*}$ University of Liège - Department of Mathematics, Grande Traverse 12 - B37, B-4000 Liège (Sart-Tilman), Belgium, P.Mathonet@ulg.ac.be 
In this paper, assuming that $\operatorname{Dim} M>1$, we compute all the linear intertwining operators

$$
\mathcal{T}: \mathcal{D}_{\lambda_{1}, \mu_{1}}^{p} \rightarrow \mathcal{D}_{\lambda_{2}, \mu_{2}}^{q},
$$

that is, the operators $\mathcal{T}$ such that

$$
L_{X}^{\lambda_{2}, \mu_{2}}(\mathcal{T}(D))=\mathcal{T}\left(L_{X}^{\lambda_{1}, \mu_{1}} D\right),
$$

for every $D \in \mathcal{D}_{\lambda_{1}, \mu_{1}}^{p}$ and every $X \in \operatorname{Vect}(M)$. This problem is a generalization of the question treated in [2] and [3] and we recover the results of these papers in the case $\lambda_{1}=\mu_{1}$ and $\lambda_{2}=\mu_{2}$. Our method is as follows: We first determine the conditions of existence of non local intertwining operators (i.e. operators that do not preserve the support of their arguments) : these are defined on the modules $\mathcal{D}_{\lambda, \lambda-1}^{0}$ and $\mathcal{D}_{0,-1}^{p}$. We describe them all and then we confine ourselves to the study of local intertwining operators. It is well-known that the restrictions of such a $\mathcal{T}$ over the relatively compact open subsets of $M$ are differential operators. We then show that if the difference of shifts $s=\left(\lambda_{2}-\mu_{2}\right)-\left(\lambda_{1}-\mu_{1}\right)$ is not equal to zero or one (in the case $\operatorname{Dim} M=2$ ), then $\mathcal{T}=0$.

In the case $s=0$, we show that $\mathcal{T}$ is filtered with respect to $p$, i.e. that if $r \leq p$, then $\mathcal{T}$ maps $\mathcal{D}_{\lambda_{1}, \mu_{1}}^{r}$ into $\mathcal{D}_{\lambda_{2}, \mu_{2}}^{r}$. We also show that $\mathcal{T}$ is completely determined by its restriction to $\mathcal{D}_{\lambda_{1}, \mu_{1}}^{2}$. We then find all the solutions for $p=2$, and those that extend to the higher order differential operators.

In the case $s=1$, we present a fundamental example of intertwining operator defined on $\mathcal{D}_{\lambda, \lambda}^{0}$ with values in $\mathcal{D}_{0,-1}^{1}$. Then we prove that the operators of this case are the compositions of this fundamental example with the intertwining operators of the case $s=0$.

\section{Polynomials associated to differential opera- tors}

We recall here how one can associate a polynomial to a differential operator. We refer to [3] for the details. In local coordinates on $M$ and simultaneous trivializations, any differential operator between the smooth sections of two vector bundles $E_{1}$ and $E_{2}$ over $M$ has a local expression of type

$$
D(f)_{x}=\sum_{|\alpha| \leq p} A_{\alpha, x}\left(D_{x}^{\alpha} f\right),
$$

where $\alpha=\left(\alpha^{1}, \ldots, \alpha^{m}\right) \in \mathbb{N}^{m}$ and $D_{x}^{\alpha} f$ denotes the partial derivative of order $\alpha$

$$
D_{x^{1}}^{\alpha^{1}} \ldots D_{x^{m}}^{\alpha^{m}} f
$$

of the local expression $f$ of the argument of $D$. Moreover the smooth map $x \mapsto A_{\alpha, x}$ takes values in the space of linear mappings between the typical fibers of the bundles. 
We define the associated polynomial in the following way :

$$
P(\xi ; f)_{x}=\sum_{|\alpha| \leq p} A_{\alpha, x}(f) \xi^{\alpha}
$$

where $\xi_{1}, \ldots, \xi_{m}$ are the components of $\xi \in T_{x}^{*} M$ in the basis $d x^{1}, \ldots, d x^{m}$ and

$$
\xi^{\alpha}=\xi_{1}^{\alpha^{1}} \ldots \xi_{m}^{\alpha^{m}} .
$$

This construction extends in an obvious way to multidifferential operators.

We will now give two examples that will be useful in further computations :

- The polynomial associated to the Lie derivative acting on $\mathcal{F}_{\lambda}$ :

$$
L^{\lambda}: \operatorname{Vect}(M) \times \mathcal{F}_{\lambda} \rightarrow \mathcal{F}_{\lambda}:(X, F) \mapsto L_{X}^{\lambda} F
$$

is

$$
P_{L^{\lambda}}(\theta, \xi ; X, f)=(\langle X, \xi\rangle-\lambda\langle X, \theta\rangle) f,
$$

where $\theta$ and $\xi$ are associated to $X$ and $f$ respectively.

- The polynomial associated to the Lie derivative acting on $\mathcal{D}_{\lambda, \mu}^{p}$ :

$$
L^{\lambda, \mu}: \operatorname{Vect}(M) \times \mathcal{D}_{\lambda, \mu}^{p} \rightarrow \mathcal{D}_{\lambda, \mu}^{p}:(X, D) \mapsto L_{X}^{\lambda, \mu} D
$$

is

$$
\begin{aligned}
P_{L^{\lambda, \mu}}(\theta, \eta ; X, P)= & \langle X, \eta\rangle P+\lambda\langle X, \theta\rangle \tau_{\theta} P-X \tau_{\theta} P \\
& +(\lambda-\mu)\langle X, \theta\rangle P
\end{aligned}
$$

where $P=P(\xi)$ denotes the polynomial associated to $D \in \mathcal{D}_{\lambda, \mu}^{p}, \tau_{\theta}$ acts on $P$ to give the polynomial

$$
\tau_{\theta} P(\xi)=P(\xi+\theta)-P(\xi)
$$

and where the product $X \tau_{\theta} P$ is that of the polynomials $\xi \mapsto\langle X, \xi\rangle$ and $\tau_{\theta} P$.

In our computations, we will use the whole polynomial, even if it depends in general both on the coordinates system and on the chosen trivializations.

Only its part of higher order $\sigma_{D}$, the symbol of $D$, is known to have an intrinsic meaning : It is indeed a smooth section of the fiber bundle

$$
\vee^{p} T M \otimes \operatorname{Hom}\left(E_{1}, E_{2}\right) .
$$

In our situation, we have for $D$ in $\mathcal{D}_{\lambda, \mu}^{p}$

$$
\sigma_{D} \in \Gamma\left(E_{p ; \mu-\lambda}\right)=\Gamma\left(\bigvee^{p} T M \otimes \Delta^{\lambda-\mu}(M)\right) .
$$

Moreover, one verifies easily that the following equality holds :

$$
\sigma_{L_{X}^{\lambda, \mu} D}=L_{X} \sigma_{D}
$$

where the Lie derivative on the right-hand side is the classical Lie derivative of the tensor product. 


\section{$3 \quad$ Non local intertwining operators}

In order to treat the question of existence of non local intertwining operators, we will determine the conditions to have the following property (1): Given $D \in \mathcal{D}_{\lambda, \mu}^{p}$ and $x \notin \operatorname{supp} D$, there exist $X_{i} \in \operatorname{Vect}(M)$ and $D_{i} \in \mathcal{D}_{\lambda, \mu}^{p}$ such that $x \notin \operatorname{supp} X_{i}$ and

$$
D=\sum_{i=1}^{n} L_{X_{i}} D_{i}
$$

In a first step (lemma's 3.1 and 3.2 ) we will use the results of [1] to obtain stronger results in most of the cases. Then we will need some topological lemma's to determine when the property (1) holds true in the remaining cases. We recall here two definitions of [1] :

Definition 3.1. Let $E$ be a vector bundle over a manifold $M$. A family $\mathcal{L}$ of differential operators from $\Gamma(E)$ into $\Gamma(E)$ is globally transitive if for every open subset $\omega$ of $M$ and every section $s$ with support in $\omega, s$ can be written

$$
s=\sum_{i=1}^{k} L_{i} s_{i}
$$

with $L_{i} \in \mathcal{L}, s_{i} \in \Gamma(E)$ and $\operatorname{supp} L_{i}, \operatorname{supp} s_{i} \subset \omega$.

Definition 3.2. A family $\mathcal{L}$ of differential operators from $\Gamma(E)$ into $\Gamma(E)$ is locally transitive if each point $x$ of $M$ has a neighborhood $\Omega$ such that (3) holds true for every subset $\omega$ of $\Omega$ and every $s$ with compact support in $\omega$, the number of terms in (3) being bounded above independently of $s$ and $\Omega$.

We have the following

Lemma 3.1. The family of Lie derivatives is locally transitive on the fiber bundle $E_{r ; q}=\vee^{r} T M \otimes \mathcal{F}_{q}$ if $r \neq 0$ or $q \neq-1$.

Proof. Let $x_{0}$ be a point of $M$ and $\left(U,\left(x^{1}, \ldots, x^{m}\right)\right)$ be a chart containing $x_{0}$ whose image is $\mathbb{R}^{m}$.

Let $u$ be a smooth section of $E_{r ; q}$ with compact support in $U$. We can consider $u$ of type $\varphi \otimes u_{0}^{\prime}$, where

$$
u_{0}^{\prime}=D_{x^{i_{1}}} \vee \cdots \vee D_{x^{i_{r}}},
$$

and $\varphi \in \mathcal{F}_{q}$ has compact support in $U$.

Using a linear change of coordinates, one can rewrite $u$ as $\varphi_{0} u_{0}$ where $\varphi_{0}$ is a smooth function with compact support in $U$ and

$$
u_{0}=\left|d x^{1} \wedge \cdots \wedge d x^{m}\right|^{-q} \otimes D_{x^{1}}^{r}
$$

If there exist smooth functions $\varphi_{i}, i \in\{1, \ldots, m\}$ with compact support in $U$ such that $\varphi_{0}=\sum_{i=1}^{n} D_{x^{i}} \varphi_{i}$, and if $\alpha$ has compact support in $U$ such that 
$\alpha=1$ on $\cup_{i=0}^{n} \operatorname{supp} \varphi_{i}$, then

$$
u=\varphi_{0} u_{0}=\sum_{i=1}^{n} L_{\alpha D_{x^{i}}}\left(\varphi_{i} u_{0}\right)
$$

It is well-known from analysis (see for instance [5]) that if $\varphi$ is a smooth function with compact support in $\mathbb{R}^{m}$ such that $\int_{\mathbb{R}^{m}} \varphi d x=0$, then $\varphi=\sum_{i=1}^{n} D_{x^{i}} \varphi_{i}$ where $\varphi_{i}$ are smooth functions with compact support in $\mathbb{R}^{m}$.

We have for any smooth function $\psi$

$$
L_{x^{i} D_{x^{i}}}\left(\psi u_{0}\right)=\left(x^{i} D_{x^{i}} \psi\right) u_{0}-\left(q+p_{i}\right) \psi u_{0},
$$

where $p_{i}$ denotes the number of times $D_{x^{i}}$ occurs in $u_{0}$.

If $q+p_{i}+1 \neq 0$,

$$
\int_{\mathbb{R}^{m}}\left[x^{i} D_{x^{i}} \psi-\left(q+p_{i}\right) \psi\right] d x=-\left(q+p_{i}+1\right) \int_{\mathbb{R}^{m}} \psi d x
$$

equals $\int_{\mathbb{R}^{m}} \varphi_{0} d x$ for a suitable choice of $\psi$. Thus

$$
\varphi_{0} u_{0}-L_{\alpha x^{i} D_{x^{i}}}\left(\psi u_{0}\right)=\beta u_{0}
$$

where $\int_{\mathbb{R}^{m}} \beta d x=0$, hence $\varphi_{0} u_{0}$ has the required form.

Here we have $p_{1}=r$ and $p_{i}=0$ for $i \neq 1$, thus the condition above can be satisfied if $q+1 \neq 0$ or $r \neq 0$.

Remark 3.1. In the construction above, all the vector fields that we use are at most linear in the coordinates on the support of their arguments.

Lemma 3.2. The family

$$
\mathcal{L}_{\lambda, \mu}^{p}=\left\{L_{X}^{\lambda, \mu}: \mathcal{D}_{\lambda, \mu}^{p} \rightarrow \mathcal{D}_{\lambda, \mu}^{p} \mid X \in \operatorname{Vect}(M)\right\}
$$

is globally transitive if $\lambda-\mu-1 \neq 0$ or $\lambda-\mu=1, p \neq 0$ and $\lambda \neq 0$.

Proof. Using Proposition 3 of [1], we only have to prove that $\mathcal{L}_{\lambda, \mu}^{p}$ is locally transitive. Let $x_{0}$ be a point of $M$ and $\left(U,\left(x^{1}, \ldots, x^{m}\right)\right)$ be a chart containing $x_{0}$ whose image is $\mathbb{R}^{m}$. Let $D$ be in $\mathcal{D}_{\lambda, \mu}^{p}$ with compact support in $\omega \subset U$. The symbol of $D$ is an element of $E_{p ; \mu-\lambda}$.

If $\mu-\lambda+1 \neq 0$, by the previous lemma, there exist $u_{i} \in E_{p ; \mu-\lambda}$ and $X_{i} \in \operatorname{Vect}(M)$ such that $\operatorname{supp} X_{i}, \operatorname{supp} u_{i} \subset \omega$ and

$$
\sigma_{D}=\sum_{i} L_{X_{i}} u_{i}
$$

We then find operators $D_{i}$ with compact support in $\omega$ such that $u_{i}=\sigma_{D_{i}}$ and the order of the operator

$$
D-\sum_{i} L_{X_{i}} D_{i}
$$


is strictly lower than the order of $D$. An easy induction allows then to conclude.

If $\lambda-\mu=1$, we can find in the same way operators $D_{i}$ and vector fields $X_{i}$ such that

$$
D-\sum_{i} L_{X_{i}} D_{i}=\pi(\varphi)
$$

where $\pi(\varphi)$ denotes the operator multiplication by $\varphi \in \mathcal{F}_{\mu-\lambda}$.

Now, if $X \in \operatorname{Vect}(M)$, we have

$$
L_{X}^{\lambda, \mu}\left(\varphi \otimes D_{x^{1}}\right)=L_{X}\left(\varphi \otimes D_{x^{1}}\right)+\lambda \pi\left(\varphi D_{x^{1}} D_{x^{i}} X^{i}\right),
$$

so that $\pi(\varphi)$ can be written as a finite sum of Lie derivatives acting on operators of order one (here we have to use vector fields whose components are polynomials of degree two in the coordinates).

It remains to look for what happens in the cases $p=0$ or $\lambda=0$, when $\lambda-\mu-1=0$. In both cases, we have to deal with operators of order zero : the multiplication by an element of $\mathcal{F}_{-1}$. We will now need some topological lemma's :

Lemma 3.3. Let $\mathcal{C}=\left(V_{i} \mid i \in N\right)$ be a countable and locally finite covering of $M$ by relatively compact open subsets.

- If $M$ is not compact, there exists an order $\psi$ on $\mathcal{C}$ such that for all $k \in \mathbb{N}$

$$
V_{\psi(k)} \cap\left(\bigcup_{\psi(i)>\psi(k)} V_{\psi(i)}\right) \neq \emptyset
$$

- If $M$ is compact, there exists an order $\psi$ on $\mathcal{C}$ such that (4) holds true for every $V$ in $\mathcal{C}$ except the last one.

Proof. The compact case seems to be well-known but we will present its proof to fix the notations in order to treat the non compact case. First, we associate a graph to the problem in a natural way :

The vertices of the graph are the elements of $\mathcal{C}$, and there is an edge between two vertices $V$ and $V^{\prime}$ if and only if $V \cap V^{\prime} \neq \emptyset$.

Secondly, we determine a tree $A$ in the graph as follows :

We take a vertex as the root and denote it by $L_{0}$, then we put

$$
L_{k}=\left\{V \in G \backslash \bigcup_{i<k} L_{i}: V \text { is connected by one edge to } L_{k-1}\right\} \text {. }
$$

If $V \in L_{k}$, there exists at least an element $V^{\prime}$ of $L_{k-1}$ and an edge between $V$ and $V^{\prime}$. To get a tree, we have to suppress some edges in such a way that each element of the level $L_{k}$ corresponds to one and only one element in $L_{k-1}$. We put $A=\cup_{k=0}^{\infty} L_{k}$. The graph $G$ is connected, since the manifold $M$ is connected and $\mathcal{C}$ is a covering. Hence we have $A=G$. 
Now let us suppose that $M$ is compact. There exists $q \in \mathbb{N}$ such that $A=\cup_{k=0}^{q} L_{k}$. We fix the order on $A$ from the leaves to the root: We construct the order $\psi$ in such a way that $L_{q}<L_{q-1}<\cdots<L_{0}$. It is then clear by the definition of the levels $L_{k}$ that the order $\psi$ fulfills the required property.

If $M$ is not compact, such a construction obviously does not work. We denote by $F B(V)$ the set of finite branches starting from $V \in A$ (a finite branch starting from $V$ is a finite connected component of the subtree of $A$ whose root is $V$, minus $V$ ). We consider $F B\left(L_{0}\right)$. This is a finite tree because each level contains a finite number of vertices. We fix the order $\psi$ on $F B\left(L_{0}\right)$, from the leaves to the root. In $L_{1}$, there exists $V \notin F B\left(L_{0}\right)$, since $A$ is not finite. We go on with $F B(V)$ and so on. We construct $\psi$ as

$$
\psi\left(F B\left(L_{0}\right)\right)<\cdots<\left\{\psi(F B(V)): V \in L_{k} \backslash \bigcup_{i<k} F B\left(L_{i}\right)\right\}<\cdots,
$$

and we get the required property.

Lemma 3.4. Let $\mathcal{C}_{1}=\left(U_{i} \mid i \in \mathbb{N}\right)$ be a locally finite covering of the manifold $M$ by connected relatively compact open subsets. If $M$ is not compact, there exists an order on $\left(U_{i} \mid i \in \mathbb{N}\right)$ and a covering $\mathcal{C}_{2}=\left(V_{i} \mid i \in \mathbb{N}\right)$ such that, for all $i \in \mathbb{N}$

- $V_{i}^{-} \subset U_{i}$,

- $U_{i} \not \subset \cup_{k \leq i} V_{k}^{-}$.

If $M$ is compact, there exists an order on $\left(U_{i} \mid i \leq N\right)$ and a covering $\mathcal{C}_{2}=$ $\left(V_{i} \mid i \leq N\right)$ such that the first property above holds true for every $i \leq N$ and the second one holds true except for $N$.

Proof. Suppose that $M$ is not compact. The existence of a covering $\mathcal{C}_{2}$ satisfying the first condition is well-known. We consider the order of lemma 3.3 for this covering (and modify the order of $\mathcal{C}_{1}$ in the same way). We will now modify the sets $V_{i}$ to satisfy the second property : If $U_{i} \subset \cup_{k \leq i} V_{k}^{-}$, there exists $m>k$ such that $V_{k} \cap V_{m} \neq \emptyset$. Hence, there exists an open subset $\omega$ such that $\omega^{-} \subset V_{k} \cap V_{m}$, then we modify $V_{1}, \ldots, V_{k}$ in $V_{1} \backslash \omega^{-}, \ldots, V_{k} \backslash \omega^{-}$. This process stabilizes, because every element of the covering $\mathcal{C}_{2}$ only intersects a finite number of other elements, and we get the required property.

The same construction yields the proof of the compact case, but it is not available for $U_{N}$.

Lemma 3.5. Let $u$ be an $m$-form with compact support in a connected open subset $\Omega$ of $\mathbb{R}^{m}$. There exist vector fields $X_{i}$ and $m$-forms $u_{i}$ with compact support in $\Omega$ such that

$$
u=\sum_{i=1}^{m} L_{X_{i}} u_{i}
$$

iff $\int_{\mathbb{R}^{m}} u=0$. 
Proof. By Poincaré's duality, there exists an $(m-1)$-form $v$ with compact support in $\Omega$ such that $u=d v$ iff $\int_{\mathbb{R}^{m}} u=0$. In this case, we have

$$
u=d v=\sum_{i=1}^{m} d\left(f_{i} d x^{1} \wedge \cdots \hat{\imath} \cdots \wedge d x^{m}\right),
$$

where the functions $f_{i}$ have compact support in $\Omega$. We set

$$
u_{i}=f_{i} d x^{1} \wedge \cdots \wedge d x^{m},
$$

and get

$$
u=\sum_{i=1}^{m} L_{D_{x^{i}}} u_{i} .
$$

If $\alpha_{i}$ has compact support in $\Omega$ and is equal to one on $\operatorname{supp} u_{i}$, then

$$
u=\sum_{i=1}^{m} L_{\alpha_{i} D_{x^{i}}} u_{i}
$$

hence the result.

Lemma 3.6. Let $u$ be in $\mathcal{F}_{-1}$ and let $x$ be a point of $M \backslash \operatorname{supp} u$.

- If $M$ is not compact, there exist vector fields $X_{i}$ and densities $u_{i}$ such that $x \notin \operatorname{supp} X_{i}$ and

$$
u=\sum_{i=1}^{m} L_{X_{i}} u_{i}
$$

- If $M$ is compact, this property holds true if we require in addition that $\int_{M} u=0$.

Proof. Let $\left(U_{i} \mid i \in \mathbb{N}\right)$ be a Palais covering of $M$ by connected and relatively compact domains of chart and $\left(V_{i} \mid i \in \mathbb{N}\right)$ be the covering constructed in lemma 3.4. For every $k \in \mathbb{N}$, there exists $\varphi_{k}$ with compact support in $U_{k}$, such that $\varphi_{k}=1$ in $V_{k}$.

If $M$ is not compact, for every $v \in \mathcal{F}_{-1}$ and $k \in \mathbb{N}$, there exists $F_{k}(v) \in \mathcal{F}_{-1}$, with compact support in $U_{k} \backslash\left(\cup_{i \leq k} V_{i}^{-} \cup\{x\}\right)$ such that

$$
\int_{M}\left(\varphi_{k} v+F_{k}(v)\right)=0 .
$$

Then we have

$$
u=u_{0}=\sum_{k=1}^{\infty} u_{k},
$$

where $u_{k}=\varphi_{k}\left(u_{0}-\sum_{i=1}^{k-1} u_{i}\right)+F_{k}\left(u_{0}-\sum_{i=1}^{k-1} u_{i}\right)$ can be written, using lemma 3.5 as

$$
\sum_{i=1}^{m} L_{X_{k, i}} u_{k, i}
$$


with $\operatorname{supp} X_{k, i} \subset U_{k} \backslash\{x\}$. We get the result by using the fact that $\left(U_{i} \mid i \in \mathbb{N}\right)$ is a Palais covering.

If $M$ is compact, we have a finite covering $\left(U_{i} \mid i \leq N\right)$. We can construct in the same way $u_{1}, \ldots, u_{N-1}$ such that

$$
u-\sum_{k=1}^{N-1} u_{k}
$$

has compact support in $V_{N}$. At this point, we can use lemma 3.5 for this density if its integral is equal to zero, and this is true since $\int_{M} u=0$.

Theorem 3.1. If the manifold $M$ is not compact, then every intertwining operator is local. If the manifold $M$ is compact, then there exist only two non-local intertwining operators :

$$
\mathcal{T}_{n \ell, 1}: \mathcal{D}_{0,-1}^{p} \rightarrow \mathcal{D}_{\mu, \mu}^{0}: D \mapsto \pi\left(\int_{M} D(1)\right)
$$

and

$$
\mathcal{T}_{n \ell, 2}: \mathcal{D}_{\lambda, \lambda-1}^{0} \rightarrow \mathcal{D}_{\mu, \mu}^{0}: \pi(\varphi) \mapsto \pi\left(\int_{M} \varphi\right) .
$$

Proof. Let $D \in \mathcal{D}_{\lambda_{1}, \mu_{1}}^{p}$ and $x \notin \operatorname{supp} D$. By Lemma's 3.2 and 3.6, there exist $X_{i} \in \operatorname{Vect}(M)$ and $D_{i} \in \mathcal{D}_{\lambda_{1}, \mu_{1}}^{p}$ such that $x \notin \operatorname{supp} X_{i}$ and $D-\sum_{i} L_{X_{i}} D_{i}=$ $\pi(u) \in \mathcal{D}_{\lambda_{1}, \mu_{1}}^{0}$. Moreover, we can require that $D-\sum_{i} L_{X_{i}} D_{i}$ vanishes if $M$ is not compact or $\lambda_{1}-\mu_{1} \neq 1$ or $p \neq 0$ and $\lambda_{1} \neq 0$. Note that in the remaining cases, $\pi(u)$ is the part of order zero of $D$ and is independent of the choice of $X_{i}$ and $D_{i}$.

We have, for any intertwining operator $\mathcal{T}: \mathcal{D}_{\lambda_{1}, \mu_{1}}^{p} \rightarrow \mathcal{D}_{\lambda_{2}, \mu_{2}}^{q}$ :

$$
\mathcal{T}(D)_{x}=\mathcal{T}(\pi(u))_{x} .
$$

Moreover, for any $X \in \operatorname{Vect}(M)$ and any $y \in M \backslash \operatorname{supp} u$, we have

$$
\left.L_{X}^{\lambda_{2}, \mu_{2}} \mathcal{T}(\pi(u))\right|_{y}=\left.\mathcal{T}\left(\pi\left(L_{X}^{-1} u\right)\right)\right|_{y}=0 .
$$

Then it is easily seen that $\mathcal{T}(\pi(u))_{x}$ is vanishing if $\lambda_{2} \neq \mu_{2}$ and there exists a locally constant function $C(x, u)$ such that $\mathcal{T}(\pi(u))_{x}=\pi(C(x, u))$ if $\lambda_{2}=\mu_{2}$.

Now for any $v \in \mathcal{F}_{-1}$ such that $x \notin \operatorname{supp} v$ we set

$$
v_{1}=\left(\int_{M} u\right) v-\left(\int_{M} v\right) u \text {. }
$$

We have $\int_{M} v_{1}=0$ and $x \notin \operatorname{supp} v_{1}$ then

$$
\left(\int_{M} u\right) \mathcal{T}(\pi(v))_{x}-\left(\int_{M} v\right) \mathcal{T}(\pi(u))_{x}=0,
$$

and there exists a constant $C^{\prime}$ such that $C(x, u)=C^{\prime} \int_{M} u$. 


\section{Local intertwining operators}

Any local intertwining operator $\mathcal{T}: \mathcal{D}_{\lambda_{1}, \mu_{1}}^{p} \rightarrow \mathcal{D}_{\lambda_{2}, \mu_{2}}^{q}$ is a differential operator. We can define as in Section 2 the polynomial $T$ associated to $\mathcal{T}$ over a chart $\left(U,\left(x^{1}, \ldots, x^{m}\right)\right)$. This is a polynomial with values in the linear applications between the spaces of polynomials associated to the elements of $\mathcal{D}_{\lambda_{1}, \mu_{1}}^{p}$ and $\mathcal{D}_{\lambda_{2}, \mu_{2}}^{q}$.

Definition 4.1. The difference of shifts $s$ of the modules $\mathcal{D}_{\lambda_{1}, \mu_{1}}^{p}$ and $\mathcal{D}_{\lambda_{2}, \mu_{2}}^{q}$ is the real number defined by

$$
s=\left(\lambda_{2}-\mu_{2}\right)-\left(\lambda_{1}-\mu_{1}\right) .
$$

If $x$ is a point of $U$, then $\left.T\right|_{x}$ belongs to the space

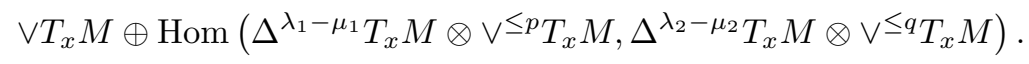

This space is isomorphic to

$$
S I\left(T_{x} M\right)=\Delta^{s} T_{x} M \otimes \vee T_{x} M \otimes \operatorname{Hom}\left(\vee^{\leq p} T_{x} M, \vee^{\leq q} T_{x} M\right) .
$$

One easily sees that the equivariance condition

$$
L_{X}^{\lambda_{2}, \mu_{2}} \mathcal{T}(D)=\mathcal{T}\left(L_{X}^{\lambda_{1}, \mu_{1}} D\right)
$$

reads, if $\left|d x^{1} \wedge \cdots \wedge d x^{m}\right|^{\lambda_{1}-\mu_{1}} \otimes P$ is associated to $D \in \mathcal{D}_{\lambda_{1}, \mu_{1}}^{p}$,

$$
\begin{gathered}
(X . T)(\eta, P)+\langle X, \eta\rangle T(\eta, P)-\langle X, \xi\rangle \tau_{\theta} T(\eta, P) \\
+\lambda_{2}\langle X, \theta\rangle \tau_{\theta} T(\eta, P)+\left(\lambda_{2}-\mu_{2}\right)\langle X, \theta\rangle T(\eta, P) \\
=\langle X, \eta\rangle T(\eta+\theta, P)-T\left(\eta+\theta, X \tau_{\theta} P\right) \\
+\lambda_{1}\langle X, \theta\rangle T\left(\eta+\theta, \tau_{\theta} P\right)+\left(\lambda_{1}-\mu_{1}\right)\langle X, \theta\rangle T(\eta+\theta, P)
\end{gathered}
$$

where $X . T$ is obtained by derivation of the coefficients of $T$ in the direction of the arbitrary vector field $X$. The terms of degree zero and one in $\theta$ of this equation yield the following

Theorem 4.1. The polynomial associated to any intertwining operator from $\mathcal{D}_{\lambda_{1}, \mu_{1}}^{p}$ to $\mathcal{D}_{\lambda_{2}, \mu_{2}}^{q}$ over every chart $\left(U,\left(x^{1}, \ldots, x^{m}\right)\right)$ has constant coefficients and is invariant under the natural action of $\mathfrak{g l}(m, \mathbb{R})$.

Proof. The term of degree zero of the above equation reads $X . T=0$. Thus, the coefficients of $T$ are constant. Hence, setting $E=\mathbb{R}^{m}, T$ belongs to the space

$$
S I(E)=\Delta^{s} E \otimes \vee E \otimes \operatorname{Hom}\left(\vee^{\leq p} E, \vee^{\leq q} E\right) .
$$

This space is isomorphic as a vector space and as a $\mathfrak{s l}(m, \mathbb{R})$-module (but not as a $\mathfrak{g l}(m, \mathbb{R})$-module) to the space of scalar polynomials :

$$
\vee E \otimes \operatorname{Hom}\left(\vee^{\leq p} E, \vee^{\leq q} E\right) .
$$


One verifies easily that the terms of degree one in $\theta$ read

$$
\begin{aligned}
& -\langle X, \xi\rangle \theta D_{\xi} T(\eta, P)-\langle X, \eta\rangle \theta D_{\eta} T(\eta, P)= \\
& -T\left(\eta, X \theta D_{\xi} P\right)+\left[\left(\lambda_{1}-\mu_{1}\right)-\left(\lambda_{2}-\mu_{2}\right)\right]\langle X, \theta\rangle T(\eta, P),
\end{aligned}
$$

where $\theta D_{\xi}$ denotes the derivation with respect to $\xi$ in the direction of $\theta$. This may be written

$$
\mathcal{L}_{X \otimes \theta} T=0
$$

where $\mathcal{L}_{X \otimes \theta}$ denotes the natural action of $X \otimes \theta \in \mathfrak{g l}(E)$ on $S I(E)$, hence the result.

Remarks : (1) It is well known that the symmetric tensors on $E$ are spanned by tensors of the type $x^{r}, x \in E$. In the sequel, we will express the invariance conditions with $P=x^{r}$ for some $x$ without loss of generality.

(2) In the sequel, using the isomorphism of the space $S I(E)$ and the space of scalar polynomials, we will only have to determine the scalar polynomial $T\left(\eta, x^{r}\right)(\xi)$.

We are now able to give some conditions on the parameters $\lambda_{1}, \mu_{1}, \lambda_{2}, \mu_{2}$ for the existence of intertwining operators :

Theorem 4.2. If $\operatorname{Dim} M>2$ and $s \neq 0$ or $\operatorname{Dim} M=2$ and $s$ is not a positive integer, then every intertwining operator from $\mathcal{D}_{\lambda_{1}, \mu_{1}}^{p}$ into $\mathcal{D}_{\lambda_{2}, \mu_{2}}^{q}$ is vanishing.

Proof. A scalar polynomial $T\left(\eta, x^{r}\right)(\xi)$ is invariant under the natural action of $\mathfrak{s l}(m, \mathbb{R})$ if and only if it has type

- $Q(\langle x, \xi\rangle,\langle x, \eta\rangle, a)$, if $m=2$;

- $Q(\langle x, \xi\rangle,\langle x, \eta\rangle)$, if $m>2$,

where $a$ is the determinant of the matrix $(\xi, \eta)$ and $Q$ is a polynomial function.

Hence, $T\left(\eta, x^{r}\right)(\xi)$ has the form $\sum_{k} a^{k} T_{k}$, where $T_{k}$ is homogeneous of degree $k$ in the variables $(\langle x, \xi\rangle,\langle x, \eta\rangle)$.

We now have to use the invariance equation with the identity matrix of $\mathfrak{g l}(m, \mathbb{R})$. This reads

$\sum_{i} \xi_{i} D_{\xi_{i}} T\left(\eta, x^{r}\right)+\sum_{i} \eta_{i} D_{\eta_{i}} T\left(\eta, x^{r}\right)=\left[r-m\left(\lambda_{1}-\mu_{1}\right)+m\left(\lambda_{2}-\mu_{2}\right)\right] T\left(\eta, x^{r}\right)$.

If $\operatorname{Dim} M>2$, then $T\left(\eta, x^{r}\right)(\xi)$ equals $T_{0}$ and the equation reads

$$
m\left[\left(\lambda_{1}-\mu_{1}\right)-\left(\lambda_{2}-\mu_{2}\right)\right] T=0 .
$$

If $\operatorname{Dim} M=2$, then we have for every $k$

$$
2 k T_{k}=2\left[\left(\lambda_{2}-\mu_{2}\right)-\left(\lambda_{1}-\mu_{1}\right)\right] T_{k},
$$

hence the result. 
Remark : When $s=0$, the scalar polynomial $T\left(\eta, x^{r}\right)(\xi)$ is invariant under the natural action of $\mathfrak{g l}(m, \mathbb{R})$. When $\operatorname{Dim} M=2$ and $s$ is a positive integer, the scalar polynomial $T\left(\eta, x^{r}\right)(\xi)$ is invariant under the natural action of $\mathfrak{s l}(2, \mathbb{R})$, and it is reduced to the monomial $a^{s} T_{s}$.

In the sequel, we will have to deal with two cases :

- $m \geq 2$ and $\left(\lambda_{1}-\mu_{1}\right)=\left(\lambda_{2}-\mu_{2}\right)$, that we call the $\mathfrak{g l}(m, \mathbb{R})$-case or classical case;

- $m=2$ and $\left(\lambda_{2}-\mu_{2}\right)-\left(\lambda_{1}-\mu_{1}\right)$ is a strictly positive integer, that we call the $\mathfrak{s l}(2, \mathbb{R})$-case.

\section{The $\mathfrak{g l}(m, \mathbb{R})$-case}

This is the case that occurs in the problem treated in [3] and [2]. The polynomial $T\left(\eta, x^{r}\right)(\xi)$ is homogeneous of degree $r$ in $(\xi, \eta)$, so it is of degree less than or equal to $r$ in $\xi$ and we have the following

Theorem 5.1. In the $\mathfrak{g l}(m, \mathbb{R})$-case, every intertwining operator respects the filtration of differential operators.

We will now consider the term of order two the equivariance equation. It reads

$$
\begin{aligned}
& \frac{1}{2}\langle X, \xi\rangle\left(\theta D_{\xi}\right)^{2} T\left(\eta, x^{r}\right)+\frac{1}{2}\langle X, \eta\rangle\left(\theta D_{\eta}\right)^{2} T\left(\eta, x^{r}\right) \\
& =\frac{1}{2} T\left(\eta, X\left(\theta D_{\xi}\right)^{2} x^{r}\right)+\theta D_{\eta} T\left(\eta, X \theta D_{\xi} x^{r}\right)-\lambda_{1}\langle X, \theta\rangle T\left(\eta, \theta D_{\xi} x^{r}\right) \\
& -\left(\lambda_{1}-\mu_{1}\right)\langle X, \theta\rangle \theta D_{\eta} T\left(\eta, x^{r}\right)+\lambda_{2}\langle X, \theta\rangle \theta D_{\xi} T\left(\eta, x^{r}\right) .
\end{aligned}
$$

The solutions of this equation are actually solutions of the whole equivariance equation since when $\operatorname{Dim} M \geq 2$, the Lie algebra of vector fields whose components are polynomials is generated by the vector fields whose components are polynomials of order at most two.

We set $T_{r}=T\left(\eta, x^{r}\right), u=\langle x, \xi\rangle$ and $v=\langle x, \eta\rangle$, then we use the following identities

$$
\begin{gathered}
T\left(\eta, X x^{i}\right)=\frac{1}{i+1}\left(X D_{x}\right) T\left(\eta, x^{i+1}\right), \\
T_{r-1}\left(\eta, X\left(\theta D_{\xi}\right)^{2} x^{r}\right)=r\langle x, \theta\rangle^{2} X D_{x} T_{r-1}
\end{gathered}
$$

and

$$
\theta D_{\eta} T_{r}\left(\eta, X \theta D_{\xi} x^{r}\right)=\langle x, \theta\rangle \theta D_{\eta} X D_{x} T_{r},
$$

to obtain an equation of the form

$$
\langle X, \xi\rangle Q_{1}+\langle X, \eta\rangle Q_{2}+\langle X, \theta\rangle Q_{3}=0 .
$$

We can consider $\langle X, \xi\rangle,\langle X, \eta\rangle$ and $\langle X, \theta\rangle$ as independent parameters since $\operatorname{Dim} M \geq 2$, and this equation is equivalent to the vanishing of the polynomials $Q_{1}, Q_{2}$ and $Q_{3}$ separately. 
So, we get the equations

$$
\begin{cases}D_{u}^{2} T_{r}-r D_{u} T_{r-1}-2 D_{u} D_{v} T_{r} & =0 \\ D_{v}^{2} T_{r}+r D_{v} T_{r-1} & =0 \\ \left(1-\lambda_{1}+\mu_{1}\right) D_{v} T_{r}+\lambda_{2} D_{u} T_{r}-r \lambda_{1} T_{r-1} & =0\end{cases}
$$

corresponding respectively to $Q_{1}, Q_{2}$ and $Q_{3}$.

\subsection{The injectivity property}

The restriction of an intertwining operator defined on $\mathcal{D}_{\lambda_{1}, \mu_{1}}^{p}$ to $\mathcal{D}_{\lambda_{1}, \mu_{1}}^{r}(r \leq p)$ is again an intertwining operator. The aim of this section is to know if an intertwining operator is completely determined by its restriction to differential operators of a certain order. We have the following result :

Theorem 5.2. Any intertwining operator is completely determined by its restriction to differential operators of order at most two.

Proof. Let $r$ be greater than 3. Let us suppose that $T_{i}=0, \forall i \in\{0, \ldots, r-1\}$, and show that $T_{r}=0$. In this situation, the equations become

$$
\begin{cases}D_{u}^{2} T_{r}-2 D_{u} D_{v} T_{r} & =0 \\ D_{v}^{2} T_{r} & =0 \\ \left(1-\lambda_{1}+\mu_{1}\right) D_{v} T_{r}+\lambda_{2} D_{u} T_{r} & =0\end{cases}
$$

The second one yields

$$
T_{r}=v T_{1}+T_{2}
$$

where $T_{1}$ et $T_{2}$ are polynomials independent of the variable $v$. Then the first one is equivalent to

$$
D_{u}^{2} T_{1}=0
$$

and

$$
D_{u}^{2} T_{2}=2 D_{u} T_{1}
$$

This implies that $T_{1}$ is at most linear with respect to $u$ and it is then at most of degree two in $(u, v)$ and has to vanish since $r \geq 3$. The last equation allows then to conclude in the same way.

We are now able to determine all the local intertwining operators. We will start by the operators defined on $\mathcal{D}_{\lambda_{1}, \mu_{1}}^{0}$, then on $\mathcal{D}_{\lambda_{1}, \mu_{1}}^{1}$ and so on.

\subsection{Operators of order zero}

In $D \in \mathcal{D}_{\lambda_{1}, \mu_{1}}^{0}$, then $D$ is the multiplication by an element of $\mathcal{F}_{\mu_{1}-\lambda_{1}}$. Theorems 4.1 and 5.1 imply that the only intertwining operators are multiples of the identity mapping. We have

$$
\mathcal{T}_{0}: \mathcal{D}_{\lambda_{1}, \mu_{1}}^{0} \rightarrow \mathcal{D}_{\lambda_{2}, \mu_{2}}^{0}: \mathcal{T}_{0}(\pi(\varphi))=\pi(\varphi)
$$




\subsection{Operators of order one}

In general, we can write

$$
T_{1}=a_{1} u+a_{2} v \text {. }
$$

The coefficients $a_{1}$ and $a_{2}$ have to fulfill the following equation :

$$
\left(1-\lambda_{1}+\mu_{1}\right) a_{2}+\lambda_{2} a_{1}-\lambda_{1} a=0 .
$$

We then get the following solutions

- If $\lambda_{1} \neq 0$,

first we have a bijective intertwining operator $\mathcal{T}_{1,1}$ whose local expression is given by

$$
\begin{aligned}
T_{1,1}(\eta, x)(\xi) & =a_{1}\langle x, \xi\rangle \\
T_{1,1}(\eta, 1)(\xi) & =a_{1} \frac{\lambda_{2}}{\lambda_{1}} .
\end{aligned}
$$

This implies

$$
\begin{aligned}
\mathcal{T}_{1,1}\left(\pi(f) \circ L_{X}^{\lambda_{1}}\right) & =\pi(f) \circ L_{X}^{\lambda_{2}} \\
\mathcal{T}_{1,1}(\pi(f)) & =\frac{\lambda_{2}}{\lambda_{1}} \pi(f),
\end{aligned}
$$

which ensures that $\mathcal{T}_{1,1}$ is globally defined.

Secondly we have a conjugation operator $\mathcal{T}_{1,2}$ whose local form is given by :

$$
\begin{aligned}
& T_{1,2}(\eta, x)(\xi)=a_{2}\langle x, \eta\rangle \\
& T_{1,2}(\eta, 1)(\xi)=a_{2} \frac{\mu_{2}-\lambda_{2}+1}{\lambda_{1}} .
\end{aligned}
$$

This implies

$$
\begin{aligned}
\mathcal{T}_{1,2}\left(\pi(f) \circ L_{X}^{\lambda_{1}}\right) & =\pi\left(L_{X}^{\mu_{2}-\lambda_{2}} f\right) \\
\mathcal{T}_{1,2}(\pi(f)) & =\frac{\mu_{2}-\lambda_{2}+1}{\lambda_{1}} \pi(f),
\end{aligned}
$$

hence $\mathcal{T}_{1,2}$ is globally defined.

Note that $\mathcal{T}_{1,1}$ is the identity mapping if $\lambda_{1}=\lambda_{2}$.

- If $\lambda_{1}=0$,

there is a third solution $\mathcal{T}_{1,3}$ defined by :

$$
\mathcal{T}_{1,3}(D)=\pi(D(1))
$$

We define $\mathcal{T}_{1,1}^{\prime}$ and $\mathcal{T}_{1,2}^{\prime}$ which are equal to $\mathcal{T}_{1,1}$ and $\mathcal{T}_{1,2}$ on operators of order one and vanish on operators of order zero and we have 


$$
\begin{aligned}
& \text { - if } \lambda_{2}=0 \text { and } \mu_{2}=-1: \mathcal{T}_{1,1}^{\prime}, \mathcal{T}_{1,2}^{\prime} \text { and } \mathcal{T}_{1,3} ; \\
& \text { - if } \lambda_{2}=0 \text { and } \mu_{2} \neq-1: \mathcal{T}_{1,1}^{\prime} \text { and } \mathcal{T}_{1,3} ; \\
& \text { - if } \lambda_{2} \neq 0: \mathcal{T}_{1,3} \text { and }\left(\lambda_{2}-\mu_{2}-1\right) \mathcal{T}_{1,1}^{\prime}+\lambda_{2} \mathcal{T}_{1,2}^{\prime} .
\end{aligned}
$$

The solutions in the case $\left(\lambda_{1}=0, \lambda_{2}=0\right)$ are the restrictions to operators of order one of solutions defined for any order. We will discuss them in section 5.5 .

\subsection{Operators of order two}

The most general expression of $T_{2}$ is given by

$$
T_{2}=a_{20} u^{2}+a_{11} u v+a_{0} v^{2} .
$$

The coefficients must fulfill the following system of equations

$$
\left\{\begin{array}{lll}
\left(1-\lambda_{2}+\mu_{2}\right) a_{2}+\lambda_{2} a_{1}-\lambda_{1} a & = & 0 \\
a_{20}-a_{11}-a_{1} & = & 0 \\
a_{02}+a_{2} & = & 0 \\
2\left(\mu_{2}-\lambda_{2}+1\right) a_{02}-2 \lambda_{1} a_{2}+\lambda_{2} a_{11} & = & 0 \\
\left(\mu_{2}-\lambda_{2}+1\right) a_{11}-2 \lambda_{1} a_{1}+2 \lambda_{2} a_{20} & = & 0
\end{array}\right.
$$

First, we have a general solution given by

$$
\left\{\begin{array}{l}
a_{20}=\lambda_{1}\left(1+\lambda_{1}+\mu_{1}\right)\left(1+\mu_{1}\right) \\
a_{11}=2 \lambda_{1}\left(\lambda_{1}-\lambda_{2}\right)\left(1+\mu_{1}\right) \\
a_{02}=-\lambda_{1} \lambda_{2}\left(\lambda_{1}-\lambda_{2}\right) \\
a_{1}=\lambda_{1}\left(1+\lambda_{2}+\mu_{2}\right)\left(1+\mu_{1}\right) \\
a_{2}=\lambda_{1} \lambda_{2}\left(\lambda_{1}-\lambda_{2}\right) \\
a=\lambda_{2}\left(1+\lambda_{1}+\mu_{1}\right)\left(1+\mu_{2}\right) .
\end{array}\right.
$$

This implies

$$
\left\{\begin{aligned}
\mathcal{T}\left(\pi(f) \circ L_{X}^{\lambda_{1}} \circ L_{Y}^{\lambda_{1}}\right)= & \lambda_{1}\left(1+\lambda_{1}+\mu_{1}\right)\left(1+\mu_{1}\right) \pi(f) \circ L_{X}^{\lambda_{2}} \circ L_{Y}^{\lambda_{2}} \\
& +\lambda_{1}\left(\lambda_{1}-\lambda_{2}\right)\left(1+\mu_{1}\right)\left[\pi(f) \circ L_{[Y, X]}^{\lambda_{2}}\right. \\
& \left.+\pi\left(L_{X} f\right) \circ L_{Y}^{\lambda_{2}}+\pi\left(L_{Y} f\right) \circ L_{X}^{\lambda_{2}}\right] \\
& -\lambda_{1} \lambda_{2}\left(\lambda_{1}-\lambda_{2}\right) \pi\left(L_{Y} L_{X} f\right) \\
\mathcal{T}\left(\pi(f) \circ L_{X}^{\lambda_{1}}\right)= & \lambda_{1}\left(1+\lambda_{2}+\mu_{2}\right)\left(1+\mu_{1}\right) \pi(f) \circ L_{X}^{\lambda_{2}} \\
& +\lambda_{1} \lambda_{2}\left(\lambda_{1}-\lambda_{2}\right) \pi\left(L_{X} f\right) \\
\mathcal{T}(\pi(f))= & \lambda_{2}\left(1+\lambda_{1}+\mu_{1}\right)\left(1+\mu_{2}\right) \pi(f),
\end{aligned}\right.
$$

which ensures that the intertwining operator is globally defined. This is always a solution but it vanishes for some values of the parameters. We can say that this is the unique solution (up to a multiplicative constant) when the system of equations has rank five. For given values of the parameters (when the system has rank less than five or in an equivalent way when the general solution vanishes), there exist additional solutions. These solutions actually extend to higher order differential operators and we will present them in the next section. 


\subsection{Higher order differential operators}

Here are the intertwining operators defined on spaces of differential operators of order greater than two.

\subsubsection{The identity mapping}

When $\lambda_{1}=\lambda_{2}$, the identity mapping is obviously an intertwining operator. Its local expression is given by

$$
T\left(\eta, x^{r}\right)(\xi)=x^{r}(\xi)
$$

\subsubsection{The projection on operators of order zero}

When $\lambda_{1}=0$, the part of order zero of a differential operator has an intrinsic meaning : it is the result of the evaluation of the operator on the constant function 1 . We can define a projection in the following way :

$$
\mathcal{T}_{p r}: \mathcal{D}_{0, \mu_{1}}^{p} \rightarrow \mathcal{D}_{\lambda_{2}, \mu_{1}+\lambda_{2}}^{0}: D \mapsto \pi(D(1)) .
$$

It is easily seen that this is an intertwining operator. Its local expression is

$$
T_{p r}\left(\eta, x^{r}\right)(\xi)=1,
$$

if $r=0$ and

$$
T_{p r}\left(\eta, x^{r}\right)(\xi)=0
$$

else.

Note that there exists a link between this operator and the ones that we have found in Theorem 3.1 : we have

$$
\mathcal{T}_{n \ell, 1}=\mathcal{T}_{n \ell, 2} \circ \mathcal{T}_{p r}
$$

\subsubsection{The bijective conjugation}

This operator has already been discussed in [3]. It exists when $\mu_{1}+\lambda_{2}=-1$ or in an equivalent way when $\mu_{2}+\lambda_{1}=-1$. In any chart domain $U$, given $f \in \mathcal{F}_{\lambda_{1}}$, $D \in \mathcal{D}_{\lambda_{1}, \mu_{1}}^{p}$ with compact support in $U$ and $g \in \mathcal{F}_{\lambda_{2}}$, we have $D(f) g \in \mathcal{F}_{-1}$ and we define $\mathcal{T}_{b c}$ by

$$
\int_{U} D(f) g=\int_{U} f \mathcal{T}_{b c}(D) g
$$

We readily get

$$
\mathcal{T}_{b c}\left(\pi(\varphi) \circ L_{X_{1}}^{\lambda_{1}} \circ \cdots \circ L_{X_{r}}^{\lambda_{1}}\right)=(-1)^{r}\left(L_{X_{r}}^{\mu_{2}} \circ \cdots \circ L_{X_{1}}^{\mu_{2}} \circ \pi(\varphi)\right) .
$$

This implies that $\mathcal{T}_{b c}$ is globally defined. Its local expression is given by

$$
T\left(\eta, x^{r}\right)(\xi)=(-1)^{r}(\langle x, \xi\rangle+\langle x, \eta\rangle)^{r} .
$$




\subsubsection{The internal conjugation}

When $\mu_{1}=-1$ and $\lambda_{2}=0$, we can compose the bijective conjugation with the projection on operators of order zero. Since we have an element of $\mathcal{D}_{0, \mu_{2}}^{0}$, we can apply $\mathcal{T}_{0}$ to suppress the condition on $\lambda_{2}$. We define

$$
\mathcal{T}_{i c}=\mathcal{T}_{0} \circ \mathcal{T}_{p r} \circ \mathcal{T}_{b c} .
$$

Its local expression is given by

$$
T_{i c}\left(\eta, x^{r}\right)(\xi)=(-1)^{r}\langle x, \eta\rangle^{r} .
$$

We call this operator internal conjugation because it transforms the derivatives of the operator in derivatives acting on the coefficients of the operator.

Now, we are able to discuss the solutions found in section 5.3, for operators or order one : we consider another basis of the linear space generated by the solutions $\mathcal{T}_{1,1}^{\prime}, \mathcal{T}_{1,2}^{\prime}$ and $\mathcal{T}_{1,3}$ and we get

- If $\lambda_{1}=0, \lambda_{2}=0$ and $\mu_{1}=-1$, we have three independent solutions given by the identity, the projection on operators of order zero, and the bijective conjugation.

- If $\lambda_{1}=0, \lambda_{2}=0$ and $\mu_{1} \neq-1$, we have two independent solutions given by the identity and the projection on operators of order zero.

Finally we have the following result that ends the study of the $\mathfrak{g l}(m, \mathbb{R})$-case :

Theorem 5.3. The previous examples are the only intertwining operators defined on $\mathcal{D}_{\lambda, \mu}^{p}$, when $p$ is strictly greater than two.

Proof. It is sufficient to solve the system of equations for the operators of order three and to use the injectivity property.

\section{The $\mathfrak{s l}(2, \mathbb{R})$-case}

We will now consider the case where the dimension of $M$ is equal to two and the difference of shifts $s$ is a strictly positive integer. The most general expression of the scalar polynomial $T\left(\eta, x^{r}\right)(\xi)$ is given by

$$
\operatorname{dtm}(\xi, \eta)^{s} T_{r}(u, v),
$$

where $T_{r}(u, v)$ is a polynomial homogeneous of degree $r$ in the variables $u$ and $v$. We will once again use the equation (5) to determine all the intertwining operators. The first theorem of this section gives necessary conditions for the existence of non vanishing intertwining operators :

Theorem 6.1. In the $\mathfrak{s l}(2, \mathbb{R})$-case, there exist non vanishing intertwining operators from $\mathcal{D}_{\lambda_{1}, \mu_{1}}^{p}$ to $\mathcal{D}_{\lambda_{2}, \mu_{2}}^{q}$ only if $\lambda_{1}=\mu_{1}, \lambda_{2}=0$ and $s=1$. 
Proof. We consider a polynomial $x^{r}$ and choose a basis such that $x=e_{1}$, then we read the equation (5) with $X=x$. The coefficient of $\theta_{2}^{2}$ readily yields

$$
\frac{s(s-1)}{2}\left(\xi_{1} \eta_{1}^{2}+\eta_{1} \xi_{1}^{2}\right) T_{r}=0,
$$

hence $s=1$.

Now if we read the equation with $X=e_{2}$ (and $\left.s=1\right)$, we get

$$
\left(\lambda_{1}-\mu_{1}\right) \xi_{1} T_{r}-\lambda_{2} \eta_{1} T_{r}=0,
$$

so that $T_{r} \neq 0$ only if $\lambda_{1}=\mu_{1}$ and $\lambda_{2}=0$ (hence $\mu_{2}=-1$ ).

We will now present the fundamental example of intertwining operator of the $\mathfrak{s l}(2, \mathbb{R})$-case.

Theorem 6.2. The operator $\mathcal{T}_{f}$ defined by :

$$
\mathcal{T}_{f}: \mathcal{D}_{\lambda, \lambda}^{0} \rightarrow \mathcal{D}_{0,-1}^{1}: \pi(\varphi) \mapsto \mathcal{T}_{f}(\pi(\varphi)): g \rightarrow|d \varphi \wedge d g|
$$

is an intertwining operator in the $\mathfrak{s l}(2, \mathbb{R})$-case.

This readily allows us to present more examples of intertwining operators in the $\mathfrak{s l}(2, \mathbb{R})$-case : if we compose an intertwining operator of the $\mathfrak{g l}(m, \mathbb{R})$ case with the fundamental example, then we still have an intertwining operator. We will prove in the next theorem that there is no other way to construct intertwining operators in the $\mathfrak{s l}(2, \mathbb{R})$-case.

Theorem 6.3. The intertwining operators of the $\mathfrak{s l}(2, \mathbb{R})$-case are $\mathcal{T}_{f}, \mathcal{T}_{f} \circ \mathcal{T}_{1,2}$, $\mathcal{T}_{f} \circ \mathcal{T}_{i c}$ and $\mathcal{T}_{f} \circ \mathcal{T}_{p r}$.

Proof. We consider an intertwining operator defined on $\mathcal{D}_{\lambda, \lambda}^{p}$. We use the remaining equation (5) with $x=X=e_{1}$, write

$$
T_{r}=\sum_{k=0}^{r} C_{k, r} u^{k} v^{r-k},
$$

and consider the coefficient of $\theta_{1} \theta_{2}$.

This yields

$$
\begin{cases}(\ell+1) C_{\ell+1, r} & =-\ell C_{\ell, r} \\ r C_{r, r} & =0,\end{cases}
$$

the first equation being available for $\ell=0, \ldots, r-1$. Hence for any $r \in\{0, \ldots, p\}$ we have $T\left(\eta, x^{r}\right)(\xi)=C_{0, r} v^{r} \mathrm{dtm}(\xi, \eta)$. This local expression fulfills the equivariance condition if and only if the coefficients $C_{0, r}$ fulfill the following equations

$$
\begin{cases}r(r-1)\left(C_{0, r}+C_{0, r-1}\right) & =0 \\ r\left(\lambda_{1} C_{0, r-1}-C_{0, r}\right) & =0\end{cases}
$$

for $r \in\{0, \ldots, p\}$, hence we get the local expressions 
- when $p=0$ :

$$
T_{0}=\operatorname{dtm}(\xi, \eta),
$$

which corresponds to $T_{f}$;

- when $p=1$ :

$$
\left\{\begin{array}{l}
T_{0}=\operatorname{dtm}(\xi, \eta) \\
T_{1}=\lambda_{1} v \operatorname{dtm}(\xi, \eta)
\end{array}\right.
$$

which corresponds to $\mathcal{T}_{f} \circ \mathcal{T}_{1,2}$;

- when $\mu_{1}=\lambda_{1}=-1$ :

$$
T_{r}=(-1)^{r} v^{r} \operatorname{dtm}(\xi, \eta)
$$

which corresponds to $\mathcal{T}_{f} \circ \mathcal{T}_{i c}$;

- when $\lambda_{1}=0$ :

$$
T_{r}=\delta_{r 0} \operatorname{dtm}(\xi, \eta),
$$

which is the local expression of $\mathcal{T}_{f} \circ \mathcal{T}_{p r}$.

Acknowledgements. The author would like to thank Professors M. de Wilde and P.B.A. Lecomte for their help during the making of this paper.

\section{References}

[1] M. De Wilde and P. B. A. Lecomte. Some characterizations of differential operators on vector bundles. In E. B. Christoffel (Aachen/Monschau, 1979), pages 543-549. Birkhäuser, Basel, 1981.

[2] C. Duval and V. Ovsienko. Space of second-order linear differential operators as a module over the Lie algebra of vector fields. Adv. Math., 132(2):316-333, 1997.

[3] P. B. A. Lecomte, P. Mathonet, and E. Tousset. Comparison of some modules of the Lie algebra of vector fields. Indag. Math. (N.S.), 7(4):461-471, 1996.

[4] J. Peetre. Une caractérisation abstraite des opérateurs différentiels. Math. Scand., 7:211-218, 1959.

[5] L. Schwartz. Théorie des distributions. Publications de l'Institut de Mathématique de l'Université de Strasbourg, No. IX-X. Nouvelle édition, entiérement corrigée, refondue et augmentée. Hermann, Paris, 1966.

[6] H. Weyl. The classical groups. Princeton Landmarks in Mathematics. Princeton University Press, Princeton, NJ, 1997. Their invariants and representations, Fifteenth printing, Princeton Paperbacks. 